\title{
Concurrent Use of Cyclin Dependent Kinase 4/6 (CDK4/6) Inhibitors with Palliative Radiotherapy for Metastatic Breast Cancer Patients: A Review of Toxicity
}

\author{
(1) Özlem SÖNMEZ,' (1) Evrim TEZCANLI²
}

'Department of Medical Oncology, Acıbadem Mehmet Ali Aydınlar University, Istanbul-Turkey

${ }^{2}$ Department of Radiation Oncology, Acıbadem Altunizade Hospital, Istanbul-Turkey

\begin{abstract}
SUMMARY
Breast cancer remains to be the most common cancer in women. Given the recent developments in oncology, while more women with early breast cancer are being cured, patients with metastatic breast cancer are living longer. There have been improvements in the treatment of hormone-sensitive metastatic breast cancer by the addition of CDK $4 / 6$ inhibitors to endocrine treatment. CDK 4 and CDK 6 control entry into the cell cycle via estrogen signaling pathway and their inhibition was shown to prevent tumor cell cycle progression. Three CDK4/6 inhibitors available for hormone receptor-positive, human epidermal growth factor receptor 2-negative metastatic breast cancer are palbociclib, ribociclib, and abemaciclib. CDK 4/6 inhibitors are suggested as radiosensitizers given their mechanism of action that inhibits cell cycle progression as well as DNA repair. However, there are concerns regarding their concurrent use with radiotherapy given their potential to delay normal tissue repair. Currently, there are several retrospective series suggesting a site-dependent toxicity. After reviewing preclinical and retrospective studies, we suggest further studies are required to clarify the safety of this combination. Until then, oncologists should evaluate each case with caution regarding the potential risks of CDK 4/6 inhibitors in combination with radiotherapy.
\end{abstract}

Keywords: CDK 4/6 inhibitors; concurrent radiotherapy; metastatic breast cancer; toxicity.

Copyright $\odot$ 2021, Turkish Society for Radiation Oncology

\section{Introduction}

Although metastatic breast cancer is unlikely to be cured, endocrine therapies (ET) with or without other systemic treatments provide longer survival for hormone-sensitive breast cancer patients.

The initial choice of treatment for hormone-sensitive, human epidermal growth factor receptor 2-negative (HER-2) negative breast cancer is ET as long as the patient is not symptomatic and there is no concern of life-threatening visceral crisis. Menopausal status, ad- juvant endocrine treatment, and the time to metastases help define the best ET option.

Several trials have proved that the addition of other agents to tamoxifen and aromatase inhibitors (AI) provided benefit when compared to ET alone. Recently published three randomized controlled trials with cyclin-dependent kinase $4 / 6$ inhibitors, palbociclib, ribociclib, and abemaciclib showed progression-free survival benefit when compared to AI alone.[1-3]

The evidence suggests synergism between CDK 4/6 inhibitors and AI or fulvestrant that inhibits cell cycle 
progress. [4] CDK 4/6 inhibitors can provide benefits for patients who developed ET resistance and they can be the treatment of choice to avoid or delay ET resistance.

Palbociclib is the first CDK 4/6 inhibitor to receive FDA approval. Approval in Europe was received after PALOMA 2 and 3 studies.[5-7] The PALOMA-3 study showed progression-free survival benefit for hormone receptor-positive, HER-2 negative metastatic breast cancer when CDK4/6 inhibitor palbociclib was combined with fulvestrant.[6,7] It is dosed as $125 \mathrm{mg} /$ day, for 3 weeks followed by a 1 week break.[1] European approval for ribociclib was received based on MONALEESA 2 and 3 trials.[8,9] Abemaciclib received European approval based on MONARCH 2 and 3 studies.[10,11] Ribociclib is used at $600 \mathrm{mg} /$ day for 3 weeks followed by a 1 week break while abemaciclib is used continuously at $150 \mathrm{mg}$, twice a day.

A meta-analysis comparing all three CDK 4/6 inhibitors in combination with an AI found no significant differences regarding the PFS benefit. Only abemaciclib was evaluated for patients with brain metastases and can be the agent of choice for this patient group.[12] Therefore, the agent of choice is based on side effects mostly. Palbociclib can cause neutropenia, while abemaciclib can cause diarrhea. Ribociclib should be used with caution when patients are using QTc prolonging agents since it can enhance this effect, it also can cause liver toxicity.

About $60 \%$ of metastatic breast cancer patients require radiotherapy. There have been conflicting literature about the safety of CDK4/6 inhibitor administration concurrent with radiotherapy and this article aims to review the literature on reported toxicities on this subject.

\section{Preclinical Studies}

Preclinical studies suggest CDK 4/6 inhibitors may create a radiosensitizer effect given their mechanism of action. First, they cause a cell cycle arrest at G1, and by inhibiting the cycle progression to radioresistant $S$ phase, CDK 4/6 inhibitors can enhance the effects of radiation. Second, they inhibit DNA double-strand break repair, which may also increase RT effectiveness as well as side effects.[13] An animal study investigating the gastrointestinal (GI) toxicity when single or fractionated radiation was applied concurrently with palbociclib.[14] The authors showed exacerbated GI toxicity when palbociclib and fractionated abdominal radiotherapy were used together.

\section{Clinical Data}

Until this date, there are only published retrospective series on the safety of CDK $4 / 6$ inhibitors concurrent use with RT. The randomized controlled MONARCH, PALOMA, and MONALEESA trial protocols did not forbid palliative radiotherapy. However, PALOMA trial protocols were the only ones concerned with concurrent treatment toxicity since they recommended to suspend palbociclib from a day earlier than the start of RT and initiate 7 days after RT.[5,6] There were only a total of 26 patients receiving palliative RT in all three PALOMA trials, and since palbociclib was not used concurrently there is no data to indicate safety or increased toxicity.

There are several retrospective studies exploring the safety of CDK 4/6 inhibitor concurrent use with RT. Although most of these studies did not report unexpected toxicity, there are several case reports suggesting higher toxicity with this combination.

The most recent retrospective analysis by Meattini et al.[15] reported the toxicity data of 85 patients receiving CDK $4 / 6$ inhibitors, $16.5 \%$ of these patients received concurrent metastases directed RT while 13\% was treated consequently. Although a substantial number of patients, $41 \%$ underwent dose reduction, and $6 \%$ discontinued due to side effects, there was no increased toxicity for either RT group when compared to no RT patients.

Kalash et al.[16] reported a three patient case series, two of these patients received lung RT while the $3^{\text {rd }}$ patient had chest wall RT. All three patients developed lung toxicity with pulmonary fibrosis and radiation pneumonitis. Patients that received lung RT had steroid-resistant pneumonitis that resolved when palbociclib was stopped.

Another case series was recently published by Van Aken et al.[17] about three patients experiencing unexpected toxicity after radiotherapy with concurrent palbociclib.One of these patients received pelvic RT for bone metastases and developed Gr 3 enterocolitis and diarrhea, while another patient receiving RT for hip developed Gr 3 edema and Gr 2 dermatitis. Third patient received $\mathrm{RT}$ to the mediastinum, although palbociclib was withheld 3-4 days prior to RT and was restarted 8-9 days after, this patient developed prolonged dysphagia with Gr 2 esophageal ulcer.

Case reports by Dasgupta et al.[18] and Kawamato et al.[19] also reported two cases of Gr 3 colitis after pelvic RT with palbociclib.

Guerini et al.[20] retrospectively analyzed 18 patients receiving palbociclib, ribociclib, or abemaciclib. Although they concluded acceptable toxicity, one patient receiving RT to L5 vertebra, sacrum, and right ischium developed Gr 3 ileitis. They reported 61\% Gr 3-4 
neutropenia during the $3^{\text {rd }}$ month after RT, however, this was comparable to CDK 4/6 inhibitors alone.

Nasir et al.[21] reported a case of Gr 3 esophagitis after RT with palbociclib, symptoms of dysphagia and odynophagia resolved after discontinuation of palbociclib.

Messer et al.[22] also reported a case with Gr 3 esophagitis and dermatitis requiring hospitalization after receiving supraclavicular lymph node radiation with concurrent palbociclib treatment.Palbociclib was suspended and RT was completed. Gr 3 skin toxicity was also reported by other case reports.[23]

Chowdhary et al.[24] analyzed 16 patients on palbociclib treatment receiving RT for the symptomatic metastases to multiple sites including bone lesions, whole-brain RT, stereotactic radiosurgery for brain metastases, and mediastinal lesions in their retrospective analysis. Only $31 \%$ of patients received concurrent palbociclib since an interval of up to 14 days was allowed before and after RT. They reported no significant increased toxicity.

Figura et al.[25] only reported on patients receiving CDK 4/6 inhibitors with stereotactic RT for brain metastases.A total of 15 patients were included, ten received palbociclib while five received abemaciclib. Their results suggested that this treatment was well tolerated.

Ippolito et al.[26] reported on 16 patients, about $30 \%$ of these patients received ablative RT for oligometastatic or oligo-progressive disease.They did not report increased toxicity despite higher doses.

\section{Discussion}

The literature review is indicative of increased toxicity profile of CDK 4/6 inhibitors when used concurrently with RT in a site-dependent manner. Although some authors reported low toxicity for palliative bone RT, others reported increased lung and GI toxicity when CDK 4/6 inhibitors were used concurrently for the chest wall, lung, and pelvic RT. Hematologic toxicities were comparable to previous reports without RT.

Studies by Figura et al.[25] and Ippolito et al. used ablative doses, however, most probably because of the limited dose to normal organs and bone marrow, there was no significant toxicity.

Although detailed treatment field information is not available for all studies, one might postulate that the treatment field size and the dose received by normal tissues might be more indicative of toxicity. There- fore, the toxicity might improve with more advanced RT techniques limiting the dose to surrounding normal tissues. It should also be noted that current metastases-directed therapies are short and therefore when in doubt CDK 4/6 inhibitors should be suspended especially in cases where GI and lung tissues are involved, as well as patients receiving RT for multiple bone lesions.

Results from preclinical and clinical studies, although controversial, indicate CDK 4/6 inhibitors can be promising for the treatment of HER $2+$ and triplenegative breast cancer subtypes, as well in the adjuvant setting of hormone-sensitive cancer.

Moreover, there are on-going trials aiming to define the use of CDK 4/6 inhibitors beyond breast cancer since genetic disruption of Cyclin D-CDK 4/6 pathway disruption is common among several cancer types. Unfortunately, early trials were not feasible in other solid tumors since they showed low therapeutic index with high toxicity rates at doses needed to inhibit CDKs. $[27,28]$ However, there is an ongoing effort to define sensitivity and resistance biomarkers to CDK 4/6 inhibitors. Current umbrella trials include multiple tumor types that fit the genetic selection criteria. As personalized treatments are improving, if these precision medicine trials are suggestive of benefit, then the use of CDK 4/6 inhibitors will not be limited to hormone-sensitive breast cancer. There will be more concerns regarding their concurrent use with metastases-directed RT. Therefore, it is important to define the criteria and limits for concurrent RT therapy.

\section{Conclusion}

The literature review suggests depending on the treatment site, lung lesions and metastases within or close by visceral tissue hold a higher toxicity risk while limited bone RT might be less risky. Therefore, it is important to make decisions based on patients' vulnerabilities as well as treatment site and dose. After reviewing preclinical and retrospective studies, we suggest further studies are required to clarify the safety of this combination. Until we gain more information from ongoing trials and retrospective analysis with higher patient numbers, we suggest withholding CDK 4/6 inhibitors concurrent use with RT when in doubt.

Peer-review: Externally peer-reviewed.

Conflict of Interest: I have no conflict of interest.

Financial Support: I have no financial support. 


\section{References}

1. Finn RS, Martin M, Rugo HS, Jones S, Im SA, Gelmon $\mathrm{K}$, et al. Palbociclib and letrozole in advanced breast cancer. N Engl J Med 2016;375(20):1925-36.

2. Hortobagyi GN, Stemmer SM, Burris HA, Yap YS, Sonke GS, Paluch-Shimon S, et al. Updated results from MONALEESA-2, a phase III trial of first-line ribociclib plus letrozole versus placebo plus letrozole in hormone receptor-positive, HER2-negative advanced breast cancer. Ann Oncol 2018;29(7):1541-7.

3. Goetz MP, Toi M, Campone M, Sohn J, Paluch-Shimon S, Huober J, et al. MONARCH 3:Abemaciclib as initial therapy for advanced breast cancer. J Clin Oncol 2017;35(32):3638-46.

4. Lapenna S, Giordano A. Cell cycle kinases as therapeutic targets for cancer. Nat Rev Drug Discov 2009;8:547-66.

5. Finn RS, Crown JP, Lang I, Boer K, Bondarenko IM, Kulyk SO, et al. The cyclin-dependent kinase 4/6 inhibitor palbociclib in combination with letrozole versus letrozole alone as first-line treatment of oestrogen receptor positive, HER2-negative, advanced breast cancer (PALOMA-1/TRIO-18): A randomised phase 2 study. Lancet Oncol 2015;16(1):25-35.

6. Cristofanilli M, Turner NC, Bondarenko I, Ro J, Im SA, Masuda N. Fulvestrant plus palbociclib versus fulvestrant plus placebo for treatment of hormonereceptor-positive, HER2-negative metastatic breast cancer that progressed on previous endocrine therapy (PALOMA-3): Final analysis of the multicentre, double-blind, phase 3 randomised controlled trial. Lancet Oncol 2016;17:425-39.

7. Turner NC, Slamon DJ, Ro J, Bondarenko I, Im SA, Masuda N. Overall survival with palbociclib and fulvestrant in advanced breast cancer. N Engl J Med 2018;379:1926-36.

8. Janni W, Alba E, Bachelot T, Diab S, Gil-Gil M, Beck TJ. First-line ribociclib plus letrozole in postmenopausal women with HR+, HER2-advanced breast cancer: Tumor response and pain reduction in the phase 3 MONALEESA-2 trial. Breast Cancer Res Treat. 2018;169:469-79.

9. Slamon DJ, Neven P, Chia S, Fasching PA, De Laurentiis $\mathrm{M}, \mathrm{Im}$ SA. Phase III randomized study of ribociclib and fulvestrant in hormone receptor-positive, human epidermal growth factor receptor 2-negative advanced breast cancer: MONALEESA-3. J Clin Oncol 2018;36:2465-72.

10. Johnston S, Martin M, Leo AD, Im SA, Awada A, Forrester T. MONARCH 3 final PFS: A randomized study of abemaciclib as initial therapy for advanced breast cancer. Npj Breast Cancer 2019;5:1-8.

11. Sledge GW, Toi M, Neven P, Sohn J, Inoue K, Pivot X. MONARCH 2: Abemaciclib in combination with fulvestrant in women with $\mathrm{HR}+/ \mathrm{HER} 2$-advanced breast cancer who had progressed while receiving endocrine therapy. J Clin Oncol 2017;35:2875-84.

12. Giuliano M, Schettini F, Rognoni C, Milani M, Jerusalem G, Bachelot T, et al. Endocrine treatment versus chemotherapy in postmenopausal women with hormone receptor-positive, HER2-negative, metastatic breast cancer: A systematic review and network meta-analysis. Lancet Oncol 2019; 20:1360.

13. Huang CY, Hsieh FS, Wang CY, Chen LJ, Chang SS, Tsai $\mathrm{MH}$, et al. Palbociclib enhances radiosensitivity of hepatocellular carcinoma and cholangiocarcinoma via inhibiting ataxia telangiectasia-mutated kinase-mediated DNA damage response. Eur J Cancer 2018;102:10-22.

14. Lee CL, Oh P, Xu ES, Ma Y, Kim Y, Daniel AR, et al. Blocking cyclin-dependent kinase 4/6 during single dose versus fractionated radiation therapy leads to opposite effects on acute gastrointestinal toxicity in mice Int J Radiat Oncol 2018;102:1569-76.

15. Meattini I, Scoccimarro E, Saieva C, Desideri I, Visani L, Dominici L. Impact of metastases directed radiation therapy on CDK4/6 inhibitors dose reduction and treatment discontinuation for metastatic HR+/HER2-breast cancer (MBC). J Clin Oncol 2020;38:562-2.

16. Kalash R, Iarrobino NA, Beriwal S, Sun M, Glaser SM, Champ CE. Palbociclib enhances pulmonary fibrosis in patients undergoing thoracic radiation therapy: $\mathrm{A}$ case series and review of the literature. Int J Radiat Oncol 2018;102:e610.

17. van Aken ES, Beeker A, Houtenbos I, Pos FJ, Linn SC, Elkhuizen $\mathrm{PH}$, et al. Unexpected toxicity of CDK4/6 inhibitor palbociclib and radiotherapy. Cancer Rep (Hoboken) 2021;2021:e1470.

18. Dasgupta A, Sahgal A, Warner E, Czarnota GJ. Safety of palbociclib concurrent with palliative pelvic radiotherapy: Discussion of a case of increased toxicity and brief review of literature. J Med Radiat Sci 2021;68:96102.

19. Kawamoto T, Shikama N, Sasai K. Severe acute radiation-induced enterocolitis after combined palbociclib and palliative radiotherapy treatment. Radiother Oncol 2019;131:240-1.

20. Guerini AE, Pedretti S, Salah E, Simoncini EL, Maddalo $\mathrm{M}$, Pegurri L, et al. A single-center retrospective safety analysis of cyclin-dependent kinase $4 / 6$ inhibitors concurrent with radiation therapy in metastatic breast cancer patients. Sci Rep 2020;10(1):13589. 
21. Nasir UM, Mozeika AM, Sayan M, Jan I, Kowal N, Haffty B, et al. Severe gastrointestinal mucositis following concurrent palbociclib and palliative radiation therapy. Anticancer Res 2020;40:5291-4.

22. Messer JA, Ekinci E, Patel TA, Teh BS. Enhanced dermatologic toxicity following concurrent treatment with palbociclib and radiation therapy: A case report. Rep Pract Oncol Radiother 2019;24:276-80.

23. Howlett S, Harvey-Jones E, Smith D, Ahmad S, Goldsmith C, Sawyer E, et al. Does concurrent use of CDK4/6 inhibitors during palliative radiotherapy increase toxicity in patients with metastatic breast cancer? Clin Oncol 2021;33:e99.

24. Chowdhary M, Sen N, Chowdhary A, Usha L, Cobleigh MA, Wang D, et al. Safety and efficacy of palbociclib and radiation therapy in patients with metastatic breast cancer: initial results of a novel combination.
Adv Radiat Oncol 2020;4:453-7.

25. Figura NB, Potluri TK, Mohammadi H, Oliver DE, Arrington JA, Robinson TJ, et al. CDK 4/6 inhibitors and stereotactic radiation in the management of hormone receptor positive breast cancer brain metastases. J Neurooncol 2019;144:583-9.

26. Ippolito E, Greco C, Silipigni S, Dell'Aquila E, Petrianni GM, Tonini G, et al. Concurrent radiotherapy with palbociclib or ribociclib for metastatic breast cancer patients: Preliminary assessment of toxicity. Breast 2019;46:70-4.

27. O'Leary B, Finn RS, Turner NC. Treating cancer with selective CDK4/6 inhibitors. Nat Rev Clin Oncol 2016;13(7):417-30.

28. Otto T, Sicinski P. Cell cycle proteins as promising targets in cancer therapy. Nat Rev Cancer 2017;17(2):93115 . 